\title{
Gut Microbiota enabled Goitered Gazelle (Gazella subgutturosa) to Adapt to Seasonal Changes
}

\author{
Wen Qin ${ }^{1,2}$, YanGan Huang ${ }^{1}$, Lei Wang1, Gonghua Lin ${ }^{1}$, Jundong Yang1,2, \\ Pengfei Song ${ }^{1,2}$, Hongmei Gao ${ }^{1,2}$, Jingjie Zhang ${ }^{1,2}$ and Tongzuo Zhang, ${ }^{1,3, *}$ \\ ${ }^{1}$ Key Laboratory of Adaptation and Evolution of Plateau Biota, Northwest Institute \\ of Plateau Biology, Chinese Academy of Sciences, Xining 810001, No. 23 Xinning \\ Road, Chengxi District, Xining, Qinghai Province, China \\ ${ }^{2}$ University of Chinese Academy of Sciences, Beijing 100049, China \\ ${ }^{3}$ Qinghai Provincial Key Laboratory of Animal Ecological Genomics, Xining \\ 810001, Qinghai Province, China
}

\begin{abstract}
A B S T R A C T
Goitered gazelle (Gazella subgutturosa) mainly live in arid and semi-arid environments. In Qinghai Province, goitered gazelle are found only in the Qaidam Basin, which shows pronounced seasonal changes ranging from cold, dry winters to short, hot summers. The gut microbiota plays a very important role in host health, immunity, and digestion. Therefore, seasonal changes in gut microbiota can affect host digestion and metabolism in ways that help the host adapt to different forage conditions. Understanding the relationship between seasonal variations in the gut microbiota and host adaptability is a good basis for the protection of the goitered gazelle. Here, we present our findings on the diversity of the gut microbiota in fecal samples from wild goitered gazelle from Qiadam Basin, Qinghai-Tibet Plateau, which are the first such data to be reported. A total of 25 phyla were found, of which the dominant phyla are Firmicutes and Bacteroidetes in both summer and winter, representing more than $90 \%$ of the overall relative abundance. Diet is a key factor in shaping the gut microbiota. Because of large differences in host diet between summer and winter, there are significant differences in microbiota community structures according to Amova ( $\left.\mathrm{SS}=0.3871 ; \mathrm{MS}=0.387 ; \mathrm{Fs}=6.856 ; P<0.001^{* *}\right)$. In winter, goitered gazelle must face cold weather as well as shortages in water and forage. To survive in a harsh environment, the relative abundance of Bacteroides, which enhances host metabolism of saccharides, increases significantly. In addition, water reabsorption was also significantly enhanced, which helps the goitered gazelles avoid dehydration in winter.
\end{abstract}

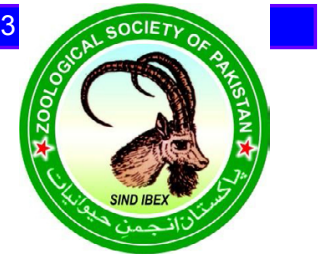

CrossMark

Article Information
Received 31 October 2018
Revised 02 January 2019
Accepted 27 January 2019
Available online 04 May 2020
Authors' Contribution
WQ and TZ designed and supervised
the study. YH, LW, JY and HG
analyzed the data. WQ, YH, GL, PS
and JZ wrote the manuscript.
Key words
Gut microbiota, Seasonal adaptation,
Seasonal microbiota change, Gazella
subgutturosa, Qaidam Basin.

Article Information

Received 31 October 2018

Revised 02 January 2019

Accepted 27 January 2019

Authors' Contribution

the study. YH, LW, JY and HG

analyzed the data. WQ, YH, GL, PS

Key words

Seasonal microbiota change, Gazella

subgutturosa, Qaidam Basin.

\section{INTRODUCTION}

G azella subgutturosa (Güldenstaedt, 1780) is also known as the goitered gazelle (IUCN SSC Antelope Specialist Group, 2017). This species generally lives in desert and semiarid habitats (Kingswood and David, 1996) and in Qinghai Province, the goitered gazelle inhabits solely the Qaidam Basin (Cai, 1989), which is an intermountain depression in the Qinghai-Tibet Plateau. The climate characteristics of the Qinghai-Tibet Plateau are cold and dry in winter, but warm and wet in summer (Liu et al., 2017), and seasonal changes in the Qaidam Basin are pronounced, with limited precipitation during the cold, long winter, and substantial rains during the warm, short summer (Shi et al., 2005). According to Xu et al. (2008),

\footnotetext{
Corresponding author: zhangtz@nwipb.cas.cn 0030-9923/2020/0005-1637 \$ 9.00/0

Copyright 2020 Zoological Society of Pakistan
}

goitered gazelle diets vary obviously across seasons, and the goitered gazelles eat noticeably fewer plant species in winter than in summer at the Kalamaili Mountain Nature Reserve. The distribution of high-quality food is scattered in arid environments (Blank et al., 2012a) and a decentralized distribution pattern in food would limit the group size of goitered gazelles to one to three individuals (Blank et al., 2012a); moreover, seasonal changes in food distribution can affect population size (Blank et al., 2012b). In 2006, Ostrowski et al. (2006) and Ostrowski and Williams (2006) demonstrated that goitered gazelles can adjust metabolic levels and change the size of their organs to survive in conditions of food and water restriction on the macro level. On the micro level, bacteria play an important role in the balance between health and disease (Lee et al., 2010). It is widely accepted that the gut microbiota can affect the host's metabolism and help meet the energy and nutritional needs of the host by improving host digestion efficiency (Nicholson et al., 2012; Sun et 
al., 2016; Tremaroli and Backhed, 2012). With a decrease in edible plant species and food quality, how do goitered gazelles face the problem of food scarcity and respond physiologically and metabolically to seasonal changes in the environment? What is the role of their microbiota in this process?

The gut microbiota can vary across seasons (Zhang et al., 2014; Maurice et al., 2015; Sun et al., 2016), and this variation may contribute to host energy metabolism according to a study on hibernating brown bears (Sommer et al., 2016). However, no reports on the seasonal variations in the gut microbiota of wild goitered gazelles. This paper presents the first profile of the gut microbiota of goitered gazelles from Qaidam Basin, Qinghai-Tibet Plateau. Significant differences in the structures of the gut microbiota were found between winter and summer, which we believe to be closely related to seasonal changes in diet. To survive in cold and dry winter, the relative abundance of Bacteroides increased significantly, moreover, the functions of saccharides metabolism enhanced significantly. Water reabsorption was also enhanced significantly in winter. We attempt to figure out the seasonal changing laws of the intestinal microbiota of wild goitered gazelles and how they enable the host to adapt to seasonal changes, including environmental and dietary changes.

The number of goitered gazelles is estimated at $120,000-140,000$ but populations throughout the range have decreased mostly because of continued illegal hunting and habitat loss. The goitered gazelle is listed as Vulnerable (IUCN SSC Antelope Specialist Group, 2017). Our data may play an important role in understanding the gut microbiota diversity and its seasonal variability, on which the viability of wild populations of goitered gazelle depend. Analysis of the changes in their intestinal microbiota can help us understand the health and nutritional status of the wild populations of goitered gazelle, which in turn will provide targeted information for their conservation.

\section{MATERIALS AND METHODS}

\section{Sample collection}

We collected 40 fresh fecal samples belonging to goitered gazelles from the same area, namely Nuomuhong Township, Qaidam Basin, in Qinghai Province. In addition, 20 samples were collected randomly in August 2016, labelled SGA1 to SGA20; and another 20 samples were collected randomly in December 2016, labelled WGA1 to WGA20. All samples were collected after natural defecation, the animals were not frightened by researchers, and no drugs were used to promote defecation. For each sample, we use disposable PE (polyethylene) gloves to avoid contamination. All samples were stored at $-20^{\circ} \mathrm{C}$ for one week after field work, then transferred to $-80^{\circ} \mathrm{C}$ storage before DNA extraction.

\section{DNA extraction and PCR amplification}

DNA from all samples was extracted using the CTAB (cetyltrimethylammonium bromide) method. To obtain $16 \mathrm{~S} \mathrm{~V} 3$ and V4 regions, target genes were amplified and using a specific barcode primer set (341F, 806R) (Berg et al., 2012; Michelsen et al., 2014). All PCR reactions were performed in a $30 \mu \mathrm{L}$ reaction mixture $(15 \mu \mathrm{L}$ Phusion Master Mix 2X, New England Biolabs; $3 \mu \mathrm{L}(6 \mu \mathrm{M})$ Primer $(2 \mu \mathrm{M}) ; 10 \mu \mathrm{L}(5-10 \mathrm{ng})$ DNA $(1 \mathrm{ng} / \mu \mathrm{L}) ; 2 \mu \mathrm{L}$ dd $\left.\mathrm{H}_{2} \mathrm{O}\right)$. Thermal cycling consisted of initial denaturation at $98^{\circ} \mathrm{C}$ for $1 \mathrm{~min}$, followed by 30 cycles of denaturation at $98^{\circ} \mathrm{C}$ for $10 \mathrm{~s}$, annealing at $50^{\circ} \mathrm{C}$ for $30 \mathrm{~s}$, and extension at $72^{\circ} \mathrm{C}$ for $30 \mathrm{~s}$, a final extension was performed at the end of the run at $72^{\circ} \mathrm{C}$ for $5 \mathrm{~min}$. We mixed an identical volume of $1 \mathrm{X}$ loading buffer with the PCR products, then performed electrophoresis on 2\% agarose gel for PCR product detection. We chose 400-450 bp bright bands for further experiments. PCR products were mixed in equidensity ratios, then extracted with GeneJET Gel Extraction Kit (Thermo Scientific).

\section{Library preparation and sequencing}

Illumina TruSeq DNA PCR-Free Library Preparation Kit (Illumina, USA) was used to generate sequence libraries according to the manufacturer's recommendations and to add index codes. We used a Qubit@2.0 Fluorometer (Thermo Scientific) and an Agilent Bioanalyzer 2100 system to assess the quality of the libraries. Once they were qualified, the library was sequenced on an Illumina HiSeq 2500 platform.

\section{Data analysis}

We used FLASH (Magoč et al., 2011) to merge the paired-end reads from the original DNA fragments according to their unique barcodes, the paired-end reads can be assigned to each sample. QIIME (Caporaso et al., 2010) was used to analyze sequences. Sequences with $\geq 97 \%$ similarity were assigned to the same OTUs. First, the reads were filtered using QIIME quality filters, then we picked a representative sequence for each OTU (Operational Taxonomic Unit) and used the RDP classifier (Wang et al., 2007) to annotate taxonomic information. We discarded all singletons whose OTUs only appeared once with Usearch and clustered the remaining OTUs by UPARSE64. Simultaneously, we were able to count the relative abundance of OTUs. Using the Mothur software, we acquired every taxonomic category information of all samples using the SILVA database. Unweighted unifrac were calculated by QIIME for Principal Coordinate Analysis (PCoA). 

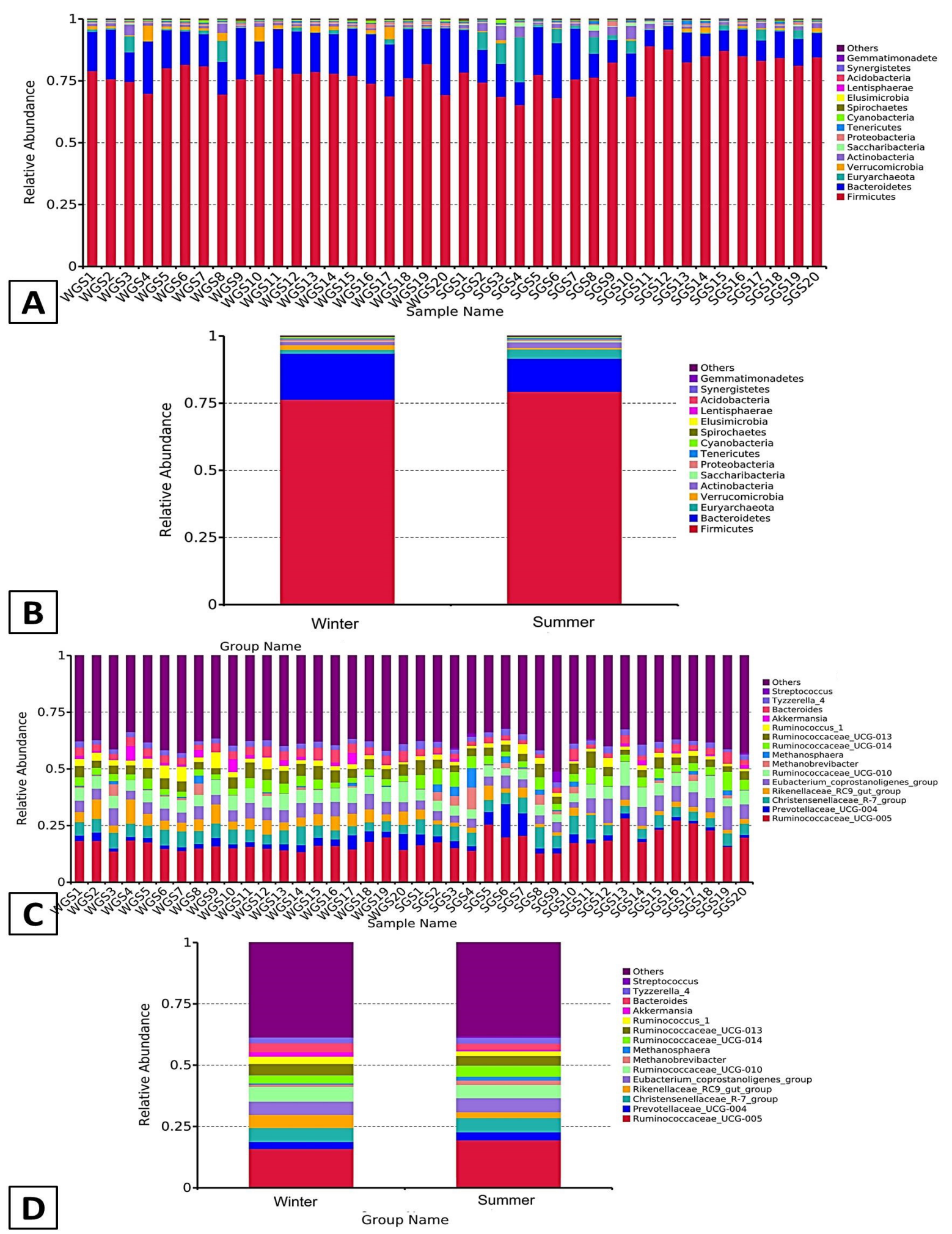

Fig. 1. A, the relative abundance of the top 15 phyla in 40 samples; $\mathbf{B}$, the relative abundance of the top 15 phyla in two groups, summer and winter; $\mathbf{C}$, the relative abundance of the top 15 genera in 40 samples; $\mathbf{D}$, the relative abundance of the top 15 genera in two groups, summer and winter. 
We commissioned Novogene Co., Ltd. to complete all experiments described in DNA extraction and PCR amplification, Library preparation and sequencing, and Data analysis.

\section{RESULTS}

\section{Data qualification}

Goods' coverage shows the sequencing depth, and indicated that our reads covered over $99 \%$ of every sample. According to effective ratio, all the above data indicate that the all the clean data generated from samples can be qualified as good data, and they were adequate to continue subsequent analysis. The details are shown in Supplementary Table I.

Using a genetic distance of $3 \%$, all $16 \mathrm{~S}$ rRNA sequence reads were assigned to OTUs and we deleted singletons to guarantee the qualification. We identified 2906 OTUs, and after removing singletons, 2754 OTUs remained. In total, 25 phyla were detected, including those that were unclassified. We classified the 25 phyla into 48 classes, 88 orders, 163 families, and 289 genera.

\section{Diversity analysis of summer and winter microbiota}

No OTUs are shared by all samples. The winter group and the summer group shared 2281 OTUs. Only 261 OTUs were specific to the summer group, while 243 OTUs were specific to the winter group. That means that the winter group and summer group are similar in OTUs composition.

At alpha diversity, the numbers of observed species, Shannon index, Simpson index, and chao 1 of the two sets of groups are shown in Table I, as estimated with R (https://www.r-project.org/). These data indicate that gut microbiota communities in the summer group are more plentiful in diversity. At the OTU level, the relative abundance of gut microbiota in the summer group is better as the chaol index shows.

Table I.- The numbers of observed species, Shannon index, Simpson index and Chao 1 between two groups.

\begin{tabular}{lcccc}
\hline Group name & Observed sp. & Shannon & Simpson & Chao1 \\
\hline Winter group & 1242.9 & 8.062777 & 0.988895 & 1318.886 \\
Summer group & 1298.3 & 7.927496 & 0.987097 & 1445.793 \\
\hline
\end{tabular}

To assess the composition of winter and summer groups, we counted the number of groups at different taxonomic levels. At the phylum level, we found a total of 25 phyla in the winter group including those that were unclassified. In the summer group, only 22 phyla were identified. Firmicutes and Bacteroidetes were the dominant phyla with relative abundance in both groups, contributing approximately $76.394 \%$ and $17.177 \%$ to the relative abundance in the winter group, and approximately $79.330 \%$ and $12.341 \%$ to the relative abundance in the summer group. Thaumarchaeota $(0.0007 \%)$, SHA$109(0.0006 \%)$, and TM6 $(0.0002 \%)$ were found only in the winter group, but their relative abundances are extremely low. The top 15 phyla from both groups are shown in Figure 1. In summer and winter, Firmicutes and Bacteroidetes were the dominant phyla in the gut microbiota communities, representing over $90 \%$ of the identical microbes. Ten phyla showed significant differences $\left(P<0.05^{*}\right)$ between the two groups according to Metastat analysis with with R (https://CRAN.R-project. org/package $=$ optparse). These are Bacteroidetes, Chlorobi, Saccharibacteria, Verrucomicrobia, Synergistetes, Tenericutes, Cyanobacteria, Gemmatimonadetes, Thaumarchaeota and Actinobacteria.

At the genus level, Ruminococcaceae UCG-005 and Ruminococcaceae UCG-010 are the dominant genera in the winter group except the unclassified cases, contributing $15.93 \%$ and $6.09 \%$ respectively to the overall relative abundance. The dominant genera are Ruminococcaceae $U C G-005$ and Christensenellaceae $R-7$ group in the summer group except the unclassified cases, contributing $19.49 \%$ and $5.80 \%$ respectively to the overall relative abundance. The relative abundance of Ruminococcaceae $U C G-005$ decreased significantly $\left(P<0.05^{*}\right)$ in winter according to Metastat analysis, while no significant differences was found in Christensenellaceae R-7_group. The top 15 genera of two groups are shown in Figure 1.



Fig. 2. Cluster analysis result of winter group and summer group according to PCoA analysis.

Seasonal variations of microbiota communities between summer and winter

We discovered a significant difference between the winter group and the summer group by Amova $\left(\mathrm{SS}=0.3871 ; \quad \mathrm{MS}=0.387 ; \quad \mathrm{Fs}=6.856 ; \quad P<0.001^{* *}\right) \quad$ with 
Mothur software. Anosim analysis with R (https://CRAN. Rproject.org/package=vegan) revealed a significant difference in the microbiota community at the OTU level between the winter group and the summer group $(\mathrm{R}=0.474$; $P=0.001)$, and our $P$ value confirmed the reliability of the data. The R-value is 0.474 , which is above 0 , indicating that inter-group differences are greater than intra-group differences. According to PCoA analysis, the two groups are well separated (Fig. 2). Based on Lefse analysis (http:// huttenhower.sph.harvard.edu/galaxy/) (LDA score is 4), we found three biomarkers (Fig. 3) showing significant differences between summer and winter groups.

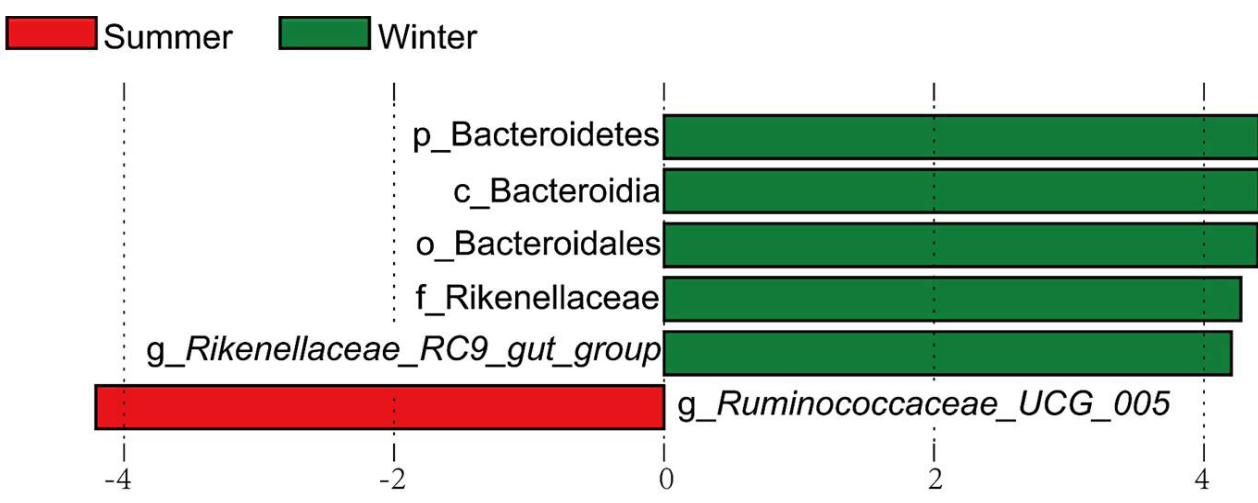

LDA SCORE $(\log 10)$

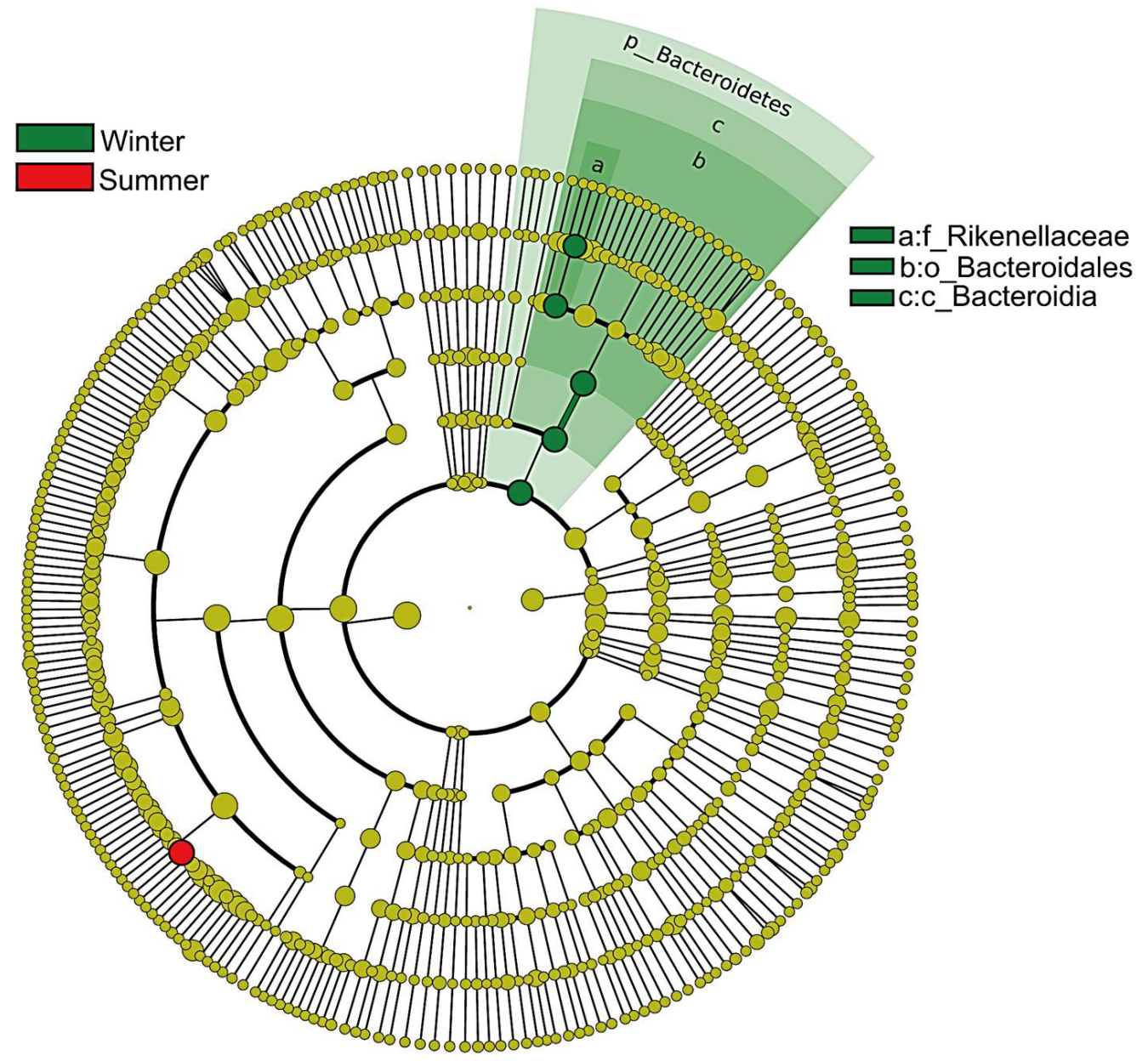

Fig. 3. Lefse analysis results of winter group and summer group (LDA score is 4). 

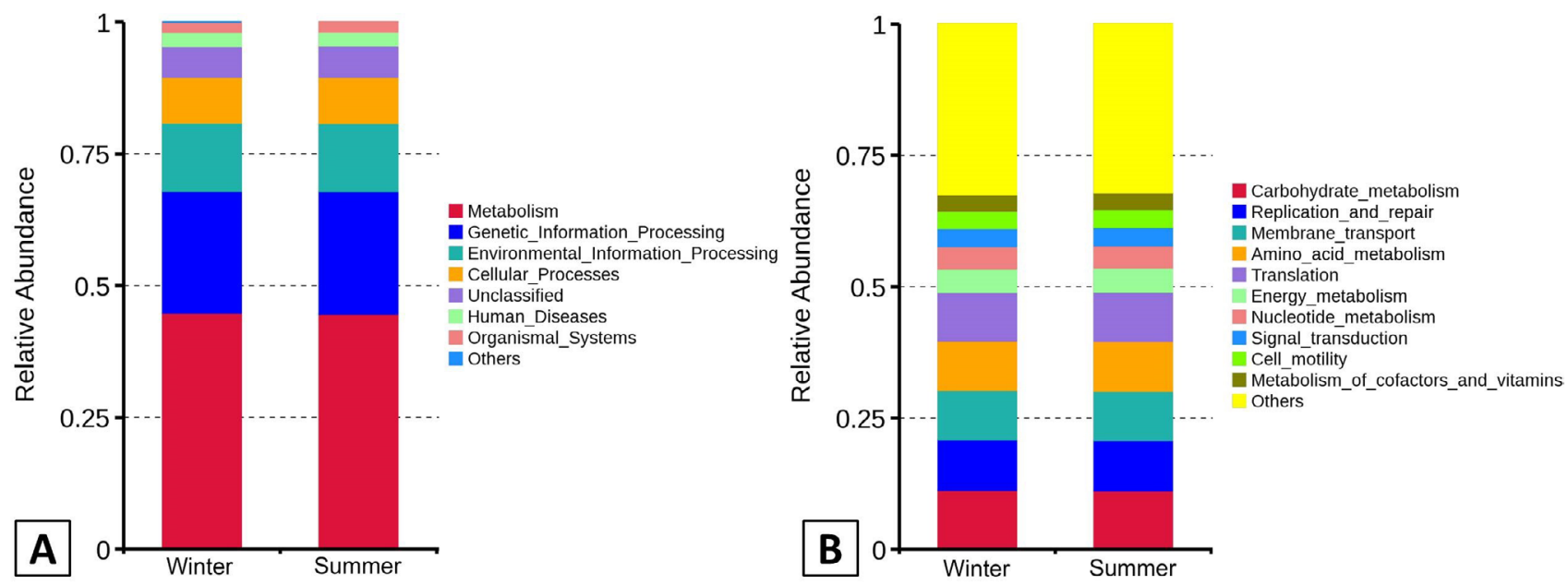

Fig. 4. Microbiota functions based on KEGG database. A, microbiota functions on first level; $\mathbf{B}$, microbiota functions on second level.

\section{Seasonal variations in gut microbiota functions}

We predicted the gut microbiota functions of winter and summer groups groups with Tax4Fun using the KEGG database (https://www.kegg.jp/) and found a significant difference $\left(p<0.05^{*}\right)$ between winter and summer groups. The main functions are transporters and two-component systems, as shown in Figure 4. The main functions that were significantly improved $\left(p<0.01^{* *}\right)$ in the winter group were galactose metabolism, lipopolysaccharide biosynthesis, carbon fixation in photosynthetic organisms, vasopressin-regulated water reabsorption, fructose and mannose metabolism, amino acid metabolism, protein digestion, and absorption. In the summer group, novobiocin biosynthesis, carbohydrate metabolism, phenylalanine metabolism, and thiamine metabolism were significantly enhanced $\left(p<0.01^{* *}\right)$.

\section{DISCUSSION}

The bacterial microbiota is commonly dominated by Firmicutes and Bacteroidetes in mammals (Bian et al., 2013; Combes et al., 2017; Huang et al., 2018; Rodriguez et al., 2015; Zhang et al., 2018). Our results are consistent with this previously published conclusion. Seasonal variation is the main reason leading to microbiota community fluctuation. Diets play an important role in shaping the microbiota (De Filippo et al., 2010). The composition and diversity of diet can affect the composition of the microbiota (Zmora et al., 2018). Xu et al. (2008) used the fecal microscopic method to evaluate the food habits of goitered gazelles (Gazella subgutturosa sairensis) from the Kalamaili Mountain Nature Reserve in northern Xinjiang, where diets varied seasonally. In winter, 17 species from six families are edible; in summer, goitered gazelle consume 30 species from twelve families (Xu et al., 2008). A healthy, diverse diet promotes a more diverse gut microbiota (Zmora et al., 2018). Goitered gazelles consume more plant species in the summer and their main foods are Ceratoides latens and some nongraminaceae species (Xu et al., 2008). Therefore, the alpha diversity of the gut microbiota is better in summer, and the seasonal variation in microbiota structures in goitered gazelle between summer and winter may be related to seasonal changes in edible plant species and food quality.

High-fat diets can cause the proportion of Ruminococcaceae to decrease and that of Rikenellaceae to increase (Daniel et al., 2014). In our research, the proportions of the relative abundance of these bacteria are also changed, Ruminococcaceae UCG-005 decreased significantly and Rikenellaceae RC9 gut group increased significantly in winter. At the family level, the relative abundance of Rikenellaceae increased significantly in winter. These changes may be due to the changes of fat content in the diet. Sacsaoul (Haloxylon ammodendron) is the main food source for goitered gazelles in winter (Xu et al., 2008), and its crude fat content increases from May to October (Gao and Ji, 1996). The changes in relative abundance of Ruminococcaceae UCG-005 and Rikenellaceae $R C 9$ gut group are likely in response to the changes in crude fat content in the goitered gazelles' diet. In addition, the content of crude fiber increases from summer to winter in sacsaoul, to help the host degrade cellulose, as a response, the relative abundance of Butyrivibrio increases significantly in winter.

Thaumarchaeota, SHA-109, and TM6 were found only in winter, and there was a significant difference in the 
relative abundance of Thaumarchaeota between summer and winter. Thaumarchaeota are capable of nitrification, reducing body odor, decreasing $\mathrm{pH}$ and removing certain nitrogen compounds (Moissl-Eichinger et al., 2017). Approximately $25 \%-45 \%$ of daily urea is degraded in the intestine in humans (Fishbach and Sonnenburg, 2011). We suppose that the main function of Thaumarchaeota in winter is to remove certain nitrogen compounds to avoid poisoning its host. Normal intestinal conditions tend to be low in $\mathrm{pH}$. Therefore, a Thaumarchaeota-induced decrease in $\mathrm{pH}$ is overall beneficial for intestinal balance. In summer, no Thaumarchaeota was found in feces, but the relative abundance of Lactobacillus increased significantly. Lactobacillus is a kind of probiotic bacteria that can also decrease intestinal $\mathrm{pH}$, thereby preventing pathogenic bacteria overgrowth and maintaining the ecological balance in the intestine (Chen and Deng, 2017). Although there is a significant difference in gut microbiota composition between summer and winter, the ultimate purpose of the favored microbes appears to be consistent, as all the changes are devoted to maintaining intestinal balance and normal metabolism.

Metabolites from intestinal microbiota are key determinants of host-microbe mutualism (Trompette et al., 2014). The temperature in the Qaidam Basin is below $0^{\circ} \mathrm{C}$ in winter, and goitered gazelles need more energy to maintain a constant body temperature. However, as the edible plants are fewer in number and their quality is poorer, it becomes more of a challenge to take in sufficient energy for survival. In our research, the relative abundance of Bacteroides increased significantly in winter, and B. thetaiotaomicron was only found in winter. Bacteroides enable the host to degrade carbohydrates, especially polysaccharides, as well as proteins, and enhance the nutrient utilization of the host (Li et al., 2017). Bacteroides are important members of the gut microbiota and Bacteroides species actively catabolize polysaccharides. Intestinal Bacteroides have evolved species-specific physical interactions with the host that mediate stable and resilient gut colonization (Wexler and Geffen, 2014). Meanwhile, galactose metabolism, lipopolysaccharide biosynthesis, fructose and mannose metabolism, and glycosaminoglycan degradation were significantly enhanced in winter. We suppose that the increase in the relative abundance of Bacteroides improves saccharide metabolism in the goitered gazelle. In winter, the enhancement of saccharide metabolism may be a way of compensating for the reduction of nutrients in foods, to ensure that there is no reduction in the total energy intake by the host. Carbohydrate metabolism in the mammalian gut may take place in a concerted manner with the help of various members of the gut microbiota. Thus, carbohydrates are presumed to be partly responsible for changes in microbiota in a changeable environment (Milani et al., 2015). It is a strategy for the goitered gazelle to regulate metabolic levels with the help of functional changes in the gut microbiota.

Qaidam Basin is surrounded by mountains that prevent warm, south-westerly air from entering the basin's interior, resulting in minimal precipitation (Wang et al., 2018). In winter, some rivers in the Qaidam Basin dry up, exposing their riverbeds. Gemmatimonadetes prefer drier soils (De Bruyn et al., 2011) and it increased significantly in winter. The changes in Gemmatimonadetes reflect an adaptation to the water shortage problem in winter faced by goitered gazelles. To survive in a water shortage environment, vasopressin-regulated water reabsorption was significantly enhanced.

\section{CONCLUSION}

Our results provide an initial view on the gut microbiota diversity of the goitered gazelle and how the microbiota enables the host to adapt to seasonal changes. We wanted to find out how the seasonal changes in the microbiota affects goitered gazelle, as well as to reveal the relationship between gut microbiota diversity and host adaptability. The goitered gazelle is the main ungulate in arid environments and its survival status is closely related to the balance of the arid landscapes. The interplay between the goitered gazelle and its gut microbiota is the result of a long evolutionary process. Understanding the relationship between the gut microbiota and the host is a precondition for conservation. Microbiota variations in the fecal matter of the goitered gazelle can be used as one of the standard tests to assess nutrient status and the animals' adaptability to environmental changes. Based on these data, a predictive diagnosis system can be established and the physical conditions of the goitered gazelle can be estimated according to the composition and functions of the gut microbiota.

\section{ACKNOWLEDGEMENTS}

We thank Dr. Rui Xing for his constructive and innovative advice. This work was supported by the Strategic Priority Research Program of the Chinese Academy of Sciences [XDA2002030302]; National Key R\&D Program of China [2017YFC0506405]; and Construction Fund for Qinghai Key Laboratories [2017ZJ-Y23].

\section{Statement on the welfare of animals}

All procedures performed in studies involving animals were approved by the Ethics and Welfare of 
Experiment Animals Committee affiliated to Northwest Institute of Plateau Biology.

\section{Supplementary material}

There is supplementary material associated with this article. Access the material online at: https://dx.doi. org/10.17582/journal.pjz/20181031121013

\section{Statement of conflict of interest}

Authors have declared no conflict of interest.

\section{REFERENCES}

Berg, J., Brandt, K.K., Al-Soud, W.A., Holm, P.E., Hansen, L.H., Sorensen, S.J. and Nybroe, O., 2012. Selection for $\mathrm{Cu}$-tolerant bacterial communities with altered composition, but unaltered richness, via long-term $\mathrm{Cu}$ exposure. Appl. environ. Microbiol., 78: 7438-7446. https://doi.org/10.1128/ AEM.01071-12

Bian, G., Ma, L., Su, Y. and Zhu, W., 2013. The microbial community in fecal samples of the White Rhinoceros (Ceratotherium simum) as determined by barcoded pyrosequencing analysis. PLoS One, $\mathbf{8}$ : 1-9. https://doi.org/10.1371/journal.pone.0070103

Blank, D., Ruckstuhl, K. and Yang, W., 2012a. Influence of population density on group sizes in goitered gazelle (Gazella subgutturosa Guld., 1780). Eur. J. Wildl. Res., 58: 981-989. https://doi.org/10.1007/ s10344-012-0641-3

Blank, D., Yang, W., Xia, C. and Xu, W., 2012b. Grouping pattern of the goitered gazelle, Gazella subgutturosa (Cetartiodactyla: Bovidae) in Kazakhstan. Mammalia, 76: 149-155. https://doi. org/10.1515/mammalia-2011-0026

Caporaso, J.G., Kuczynski, J., Stombaugh, J., Bittinger, K., Bushman, F.D., Costello, E.K., Fierer, N., Pena, A.G., Goodrich, J.K., I.Gordon, J., Huttley, G.A., Kelley, S.T., Knights, D., Koenig, J.E., Ley, R.E., Lozupone, C.A., McDonald, D., Muegge, B.D., Pirrung, M., Reeder, J., Sevinsky, J.R., Turnbaugh, P.J., Walters, W.A., Widmann, J., Yatsunenko, T., Zaneveld, J. and Knight, R., 2010. QIIME allows analysis of high-throughput community sequencing data. Nature Methods, 7: 335-336. https://doi. org/10.1038/nmeth.f.303

Cai, G., 1989. Artiodactyla. In: Economic animals in Qinghai Province (ed. D. Li). Qinghai People's Publishing House, Xining, pp. 650-651.

Chen, Z. and Deng, J., 2017. Effects of composite antibacteria peptide on intestinal microflora and $\mathrm{pH}$ value of weaning piglets. Anim. Husband. Vet.
Med., 49: 109-113.

Combes, S., Massip, K., Martin, O., Furbeyre, H., Cauquil, L., Pascal, G., Bouchez, O., Floc'h, N.L., Zemb, O., Oswald, I.P. and Gidenne, T., 2017. Impact of feed restriction and housing hygiene conditions on specific and inflammatory immune response, the cecal bacterial community and the survival of young rabbits. Animal: An Int. J. Anim. Biosci., 11: 854-863. https://doi.org/10.1017/ S1751731116002007

Daniel, H., Gholami, A.M., Berry, D., Desmarchelier, C., Hahne, H., Loh, G., Mondot, S., Lepage, P., Rothballer, M., Walker, A., Bohm, C., Wenning, M., Wagner, M., Blaut, M., Schmitt-Kopplin, P., Kuster, B., Haller, D. and Clavel, T., 2014. Highfat diet alters gut microbiota physiology in mice. The ISME J., 8: 295-308. https://doi.org/10.1038/ ismej.2013.155

De Bruyn, J.M., Nixon, L.T., Fawaz, M.N., Johnson, A.M. and Radosevich, M., 2011. Global biogeography and quantitative seasonal dynamics of Gemmatimonadetes in soil. Appl. environ. Microbiol., 77: 6295-6300. https://doi.org/10.1128/ AEM.05005-11

De Filippo, C., Cavalieri, D., di Paola, M., Ramazzotti, M., Poullet, J.B., Massart, S., Collini, S., Pieraccini, G. and Lionetti, P., 2010. Impact of diet in shaping gut microbiota revealed by a comparative study in children from Europe and rural Africa. Proc. natl. Acad. Sci. U.S.A., 107: 14691-14696. https://doi. org/10.1073/pnas.1005963107

Fischbach, M.A. and Sonnenburg, J.L., 2011. Eating for two: How metabolism establishes interspecies interactions in the gut? Cell Host Microbe, 10: 336347. https://doi.org/10.1016/j.chom.2011.10.002

Gao, W. and Ji, L., 1996. An analysis of the nutrient composition of five species of sandy plants growing in west Inner Mongonia. J. Inner Mongonia Inst. Argric. Anim. Husband., 17: 23-28.

IUCN SSC Antelope Specialist Group, 2017. Gazella subgutterosa. The IUCN red list of threatened species.

Huang, Q., Holman, D.B., Alexander, T., Hu, T., Jin, L., Xu, Z., McAllister, T.A., Acharya, S., Zhao, G. and Wang, Y., 2018. Fecal microbiota of lambs fed purple prairie clover (Dalea purpurea Vent.) and alfalfa (Medicago sativa). Arch. Microbiol., 200: 137-145. https://doi.org/10.1007/s00203-0171427-5

Kingswood, S.C. and David, B., 1996. Gazella subgutturosa. Mammalia Sp., 518: 1-10. https://doi. org/10.2307/3504145 
Lee, Y.K. and Mazmanian, S.K., 2010. Has the microbiota played a critical role in the evolution of the adaptive immune system. Science, 330: 17681773. https://doi.org/10.1126/science. 1195568

Li, Y., Hu, X., Yang, S., Zhou, J., Zhang, T., Qi, L., Sun, X., Fan, M., Xu, S., Cha, M., Zhang, M., Lin, S., Liu, S. and Hu, D., 2017. Comparative analysis of the gut microbiota composition between captive and wild forest musk deer. Front. Microbiol., 8: 1705. https://doi.org/10.3389/fmicb.2017.01705

Liu, S., Cheng, F., Dong, S., Zhao, H., Hou, X. and Wu, X., 2017. Spatiotemporal dynamics of grassland aboveground biomass on the Qinghai-Tibet Plateau based on validated MODIS NDVI. Scient. Rep., 7: 4182. https://doi.org/10.1038/s41598-017-04038-4

Magoč, T. and Salzberg, S.L., 2011. FLASH: Fast length adjustment of short reads to improve genome assemblies. Bioinformatics, 27: 2957-2963. https:// doi.org/10.1093/bioinformatics/btr507

Maurice, C.F., Knowles, S.C., Ladau, J., Pollard, K.S., Fenton, A., Pedersen, A.B. and Turnbaugh, P.J., 2015. Marked seasonal variation in the wild mouse gut microbiota. The ISME J., 9: 2423-2434. https:// doi.org/10.1038/ismej.2015.53

Michelsen, C.F., Pedas, P., Glaring, M.A., Schjoerring, J.K. and Stougaard, P., 2014. Bacterial diversity in Greenlandic soils as affected by potato cropping and inorganic versus organic fertilization. Polar Biol., 37: 61-71. https://doi.org/10.1007/s00300013-1410-9

Milani, C., Lugli, G.A., Duranti, S., Turroni, F., Mancabelli, L., Ferrario, C., Mangifesta, M., Hevia, A., Viappiani, A., Scholz, M., Arioli, S., Sanchez, B., Lane, J., Ward, D.V., Hickey, R., Mora, D., Segata, N., Margolles, A., van Sinderen, D. and Ventura, M., 2015. Bifidobacteria exhibit social behavior through carbohydrate resource sharing in the gut. Scient. Rep., 5: 15782. https:// doi.org/10.1038/srep15782

Moissl-Eichinger, C., Probst, A.J., Birarda, G., Auerbach, A., Koskinen, K., Wolf, P. and Holman, H.N., 2017. Human age and skin physiology shape diversity and abundance of Archaea on skin. Scient. Rep., 7: 4039. https://doi.org/10.1038/s41598-01704197-4

Nicholson, J.K., Holmes, E., Kinross, J., Burcelin, R., Gibson, G., Jia, W. and Pettersson, S., 2012. Host-gut microbiota metabolic interactions. Science, 336: 1262-1267. https://doi.org/10.1126/ science. 1223813

Ostrowski, S. and Joseph, W., 2006. Heterothermy of free-living Arabian sand gazelles (Gazella subgutturosa marica) in a desert environment. $J$. exp. Biol., 209: 1421-1429. https://doi.org/10.1242/ jeb.02151

Ostrowski, S., Mesochina, P. and Williams, J.B., 2006. Physiological adjustments of sand gazelles (Gazella subgutturosa) to a boom-or-bust economy: Standard fasting metabolic rate, total evaporative water loss, and changes in the sizes of organs during food and water restriction. Physiol. Biochem. Zool., 79: 810819. https://doi.org/10.1086/504614

Rodriguez, C., Taminiau, B., Brevers, B., Avesani, V., Broeck, J.V., Leroux, A., Gallot, M., Bruwier, A., Amory, H., Delmee, M. and Daube, G., 2015. Faecal microbiota characterisation of horses using 16 rDNA barcoded pyrosequencing, and carriage rate of clostridium difficile at hospital admission. BMC Microbiol., 15: 1-14. https://doi.org/10.1186/ s12866-015-0514-5

Shi, X., Zhao, Y., Dai, S., Xu, L., L, Y., Jia, H. and Zhang, Q., 2005. Research on climate change of Qaidam Basin since 1961. J. Desert Res., 25: 123-128.

Sommer, F., Stahlman, M., Ilkayeva, O., Arnemo, J.M., Kindberg, J., Josefsson, J., Newgard, C.B., Frobert, O. and Backhed, F., 2016. The gut microbiota modulates energy metabolism in the hibernating brown bear ursus arctos. Cell Rep., 14: 1655-1661. https://doi.org/10.1016/j.celrep.2016.01.026

Sun, B., Wang, X., Bernstein, S., Huffman, M.A., Xia, D.P., Gu, Z., Chen, R., Sheeran, L.K., Wagner, R.S. and Li, J., 2016. Marked variation between winter and spring gut microbiota in free-ranging Tibetan Macaques (Macaca thibetana). Scient. Rep., 6: 26035. https://doi.org/10.1038/srep26035

Tremaroli, V. and Bäckhed, F., 2012. Functional interactions between the gut microbiota and host metabolism. Nature, 489: 242-249. https://doi. org/10.1038/nature 11552

Trompette, A., Gollwitzer, E.S., Yadava, K., Sichelstiel, A.K., Sprenger, N., Ngom-Bru, C., Blanchard, C., Junt, T., Nicod, L.P., Harris, N.L. and Marsland, B.J., 2014. Gut microbiota metabolism of dietary fiber influences allergic airway disease and hematopoiesis. Nat. Med., 20: 159-166. https://doi. org/10.1038/nm.3444

Wang, Q., Garrity, G.M., Tiedje, J.M. and Cole, J.R., 2007. Naive Bayesian classifier for rapid assignment of rRNA sequences into the new bacterial taxonomy. Appl. environ. Microbiol., 73: 5261-5267. https://doi.org/10.1128/AEM.0006207

Wang, X., Kong, F., Kong, W. and Xu, W., 2018. Edaphic characterization and plant zonation in the 
Qaidam Basin, Tibetan Plateau. Scient. Rep., 8: 1822. https://doi.org/10.1038/s41598-018-20163-0

Wexler, H.M. and Geffen, D., 2014. The genus Bacteroides. In: The prokaryotes other major lineages of bacteria and the archaea (ed. E. Rosenberg). Volume 33: The genus Bacteroides, $4^{\text {th }}$ edn. Springer, Verlag, Berlin, Heidelberg, pp. 459484. https://doi.org/10.1007/978-3-642-389542_129

Xinghe, S., Yangning, Z., Sheng, D., Liang, X., Yingye, L., Hongli, J. and Qingmei, Z., 2005. Research on climate change of Qaidam Basin since 1961. J. Desert Res., 25: 123-128.

Xu, W., Fang, Q., Liu, W. and Yang, W., 2008. Food habits of goitered gazelles (Gazella subgutturosa sairensis) in northern Xinjiang. Acta Theriol. Sin., 28: $280-286$.

Zhang, J., Guo, Z., Lim, A.A.Q., Zheng, Y., Koh, E.Y., Ho, D., Qiao, J., Huo, D., Hou, Q., Huang,
W., Wang, L., Javzandulam, C., Narangerel, C., Jirimutu, Menghebilige, Lee, Y.K. and Heping, Z., 2014. Mongolians core gut microbiota and its correlation with seasonal dietary changes. Scient. Rep., 4: 1-11.

Zhang, Z., Wang, X., Han, S., Liu, C. and Liu, F., 2018. Effect of two seaweed polysaccharides on intestinal microbiota in mice evaluated by illumina PE250 sequencing. Int. J. biol. Macromol., 112: 796-802. https://doi.org/10.1016/j.ijbiomac.2018.01.192

Zhanghua, C. and Junliang, D., 2017. Effects of composite antibacteria peptide on intestinal microflora and $\mathrm{pH}$ value of weaning piglets. Anim. Husband. Vet. Med., 49: 109-113.

Zmora, N., Suez, J. and Elinav, E., 2018. You are what you eat: Diet, health and the gut microbiota. Nat. Rev. Gastroenterol. Hepatol., 16: 35-56. https://doi. org/10.1038/s41575-018-0061-2 